J. Appl. Numer. Optim. 3 (2021), No. 3, pp. 521-531

Available online at http://jano.biemdas.com

https://doi.org/10.23952/jano.3.2021.3.07

\title{
A NOTE ON MINIMAX OPTIMIZATION PROBLEMS WITH AN INFINITE NUMBER OF CONSTRAINTS
}

\author{
KWAN DEOK BAE ${ }^{1}$, TATIANA SHITKOVSKAYA ${ }^{2}$, DO SANG KIM ${ }^{1, *}$ \\ ${ }^{1}$ Department of Applied Mathematics, Pukyong National University, Busan 48513, Korea \\ ${ }^{2}$ Digital Twin Department, UNOMIC Ltd., Busan 48726, Korea
}

\begin{abstract}
In this paper, employing some advanced tools of variational analysis and generalized differentiation, we establish necessary conditions for locally optimal solutions of minimax optimization problems with infinitely many constraints. Sufficient conditions for such solutions to the considered problem are also provided by introducing generalized convex functions defined in terms of the limiting subdifferential for locally Lipschitz functions. In addition, some duality results for minimax optimization problems with infinitely many constraints are also provided. Furthermore, we derive necessary and sufficient conditions for a weak Pareto solution to the multiobjective optimization problem.
\end{abstract}

Keywords. Duality; Limiting subdifferential; Minimax problem; Optimality conditions; Semi-infinite optimization.

\section{INTRODUCTION}

Let $X$ denote the Asplund space (i.e., Banach spaces whose separable subspaces have separable duals), $\Omega$ be a nonempty locally closed subset of $X$, and let $T$ be an arbitrary (possibly infinite) index set. Consider the following minimax optimization problem

$$
\min _{x \in F} \max _{k \in K} f_{k}(x),
$$

where the feasible set $F:=\left\{x \in \Omega \mid g_{t}(x) \leq 0, t \in T\right\}$ is defined by an infinite number of inequality constraints, and the functions $f_{k}, k \in K:=\{1, \ldots, l\}$, and $g_{t}, t \in T$ are locally Lipschitz on $X$. Hence, problem $(\mathrm{P})$ becomes a minimax semi-infinite optimization problem. Hereafter, we use the notation $f:=\left(f_{1}, \ldots, f_{l}\right)$, and $g_{T}:=\left(g_{t}\right)_{t \in T}$. In what follows, $\Omega$ is always assumed to be SNC at the point under consideration. Fundamental theoretical aspects and a wide range of applications of the semi-infinite optimization have been studied intensively by many researchers recently; see, e.g., $[1,2,3,4,5,6,7]$ and the references therein. Minimax optimization problems have been the subject of immense interest in the past few years. Recently, many researchers studied optimality conditions and duality theorems for minimax optimization problems; see, e.g., $[8,9,10]$ and the references therein.

\footnotetext{
${ }^{*}$ Corresponding author.

E-mail addresses: bkduck106@naver.com (K.D. Bae), tatiana.sksy@unomic.com (T. Shitkovskaya), dskim@ pknu.ac.kr (D.S. Kim).

Received October 10, 2021; Accepted December 2, 2021.
}

(C)2021 Journal of Applied and Numerical Optimization 
In this paper, we focus on the theoretical aspects for problem $(\mathrm{P})$. By employing some advanced tools of variational analysis and generalized differentiation, we establish necessary conditions for locally optimal solutions of problem (P), under the constraint qualification (CQ). Then, we present sufficient conditions for such solutions for problem (P), by means of introducing generalized convex functions defined in terms of the limiting subdifferential for locally Lipschitz functions. In addition, we introduce a dual problem to the primal one and examine weak, strong and converse duality relations under generalized convexity assumptions. Finally, as an application, we obtain optimality conditions for a weak Pareto solution of a class of semiinfinite multiobjective optimization problems by the obtained results for problem $(\mathrm{P})$.

The rest of the paper is organized as follows. Section 2 provides some preliminaries. In Section 3, we present some results on semi-infinite minimax optimization problems, including necessary conditions for locally optimal solutions and sufficient conditions for globally optimal solutions. Section 4 is devoted to investigating duality relations for semi-infinite minimax optimization problems. The applications are given in Section 5.

\section{PRELIMinARIES}

In this section, we use the following notations and preliminary results that will be used throughout the paper; see, e.g., [11,12]. Unless otherwise specified, all spaces under consideration are assumed to be Asplund. The canonical pairing between space $X$ and its topological dual $X^{*}$ is denoted by $\langle\cdot, \cdot\rangle$, while the symbol $\|\cdot\|$ stands for the norm in the considered space. As usual, the polar cone of a set $\Omega \subset X$ is defined by

$$
\Omega^{\circ}:=\left\{x^{*} \in X^{*} \mid\left\langle x^{*}, x\right\rangle \leq 0 \text { for all } x \in \Omega\right\} .
$$

Given a multifunction $\Psi: X \rightrightarrows X^{*}$, we denote by

$\underset{x \rightarrow \bar{x}}{\operatorname{Limsup}} \Psi(x):=\left\{x^{*} \in X^{*} \mid \exists x_{k} \rightarrow \bar{x}\right.$ and $x_{k}^{*} \stackrel{w^{*}}{\rightarrow} x^{*}$ with $x_{k}^{*} \in \Psi\left(x_{k}\right)$ for all $\left.k \in \mathbb{N}:=\{1,2, \ldots\}\right\}$

the Painlevé-Kuratowski upper/outer limit of $\Psi$ at $\bar{x}$, where the notation $\stackrel{w^{*}}{\rightarrow}$ indicates the convergence in the weak* topology of $X^{*}$.

Given $\Omega \subset X$, and $\bar{x} \in \Omega$, define the Fréchet/regular normal cone to $\Omega$ at $\bar{x}$ by

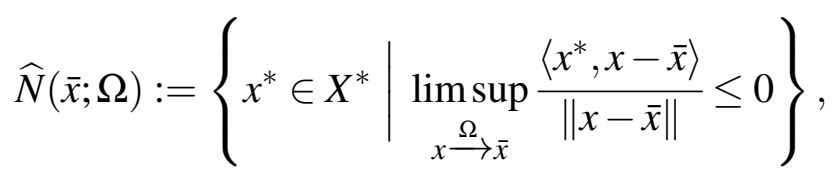

where $x \stackrel{\Omega}{\rightarrow} \bar{x}$ means that $x \rightarrow \bar{x}$ with $x \in \Omega$. If $\bar{x} \notin \Omega$, we put $\widehat{N}(\bar{x} ; \Omega):=\emptyset$.

The Mordukhovich/limiting normal cone $N(\bar{x} ; \Omega)$ to $\Omega$ at $\bar{x} \in \Omega \subset X$ is obtained from regular normal cones by taking the sequential Painlevé-Kuratowski upper limits as

$$
N(\bar{x} ; \Omega):=\operatorname{Limsup}_{x \stackrel{\Omega}{\rightarrow}_{\bar{x}}} \widehat{N}(x ; \Omega) .
$$

If $\bar{x} \notin \Omega$, we put $N(\bar{x} ; \Omega):=\emptyset$.

For an extended real-valued function $f: X \rightarrow \overline{\mathbb{R}}:=[-\infty, \infty]$, its epigraph is defined by

$$
\text { epi } f:=\{(x, \mu) \in X \times \mathbb{R} \mid \mu \geq f(x)\} \text {. }
$$


The Mordukhovich/limiting subdifferential of $f$ at $\bar{x} \in X$ with $|f(\bar{x})|<\infty$ is defined by

$$
\partial f(\bar{x}):=\left\{x^{*} \in X^{*} \mid\left(x^{*},-1\right) \in N((\bar{x}, f(\bar{x})) \text {; epi } f)\right\} .
$$

If $|f(\bar{x})|=\infty$, then one puts $\partial f(\bar{x}):=\emptyset$.

Considering the indicator function $\delta(\cdot ; \Omega)$ defined by $\delta(x ; \Omega):=0$ for $x \in \Omega$ and by $\delta(x ; \Omega):=$ $\infty$ otherwise, we have a relation between the Mordukhovich normal cone and the limiting subdifferential of the indicator function as follows (see [11, Proposition 1.79]):

$$
\partial \delta(\bar{x} ; \Omega)=N(\bar{x} ; \Omega) \text { for all } \bar{x} \in \Omega .
$$

The nonsmooth version of Fermat's rule is formulated as follows (see [11, Proposition 1.114]): If $\bar{x} \in X$ is a local minimizer of $f: X \rightarrow \overline{\mathbb{R}}$, then

$$
0 \in \partial f(\bar{x})
$$

A set $\Omega \subset X$ is sequentially normally compact (SNC) at $\bar{x} \in \Omega$ if for any sequences

$$
x_{k} \stackrel{\Omega}{\rightarrow} \bar{x} \text { and } x_{k}^{*} \stackrel{\omega^{*}}{\rightarrow} 0 \text { with } x_{k}^{*} \in \widehat{N}\left(x_{k} ; \Omega\right),
$$

one has $\left\|x_{k}^{*}\right\| \rightarrow 0$ as $k \rightarrow \infty$. Obviously, this SNC property is automatically satisfied in finite dimensional spaces.

A function $\psi: X \rightarrow \mathbb{R}$ is said to be sequentially normally compact (SNC) at $\bar{x} \in X$ if gph $\psi$ is SNC at $(\bar{x}, \psi(\bar{x}))$. According to [11, Corollary 1.69(i)], $\psi$ is SNC at $\bar{x} \in X$ if it is Lipschitz continuous around $\bar{x}$.

Lemma 2.1. [12, Theorem 2.16] Let $\Omega_{1}, \Omega_{2} \subset X$ be such that $\bar{x} \in \Omega_{1} \cap \Omega_{2}$, and that at least one of $\left\{\Omega_{1}, \Omega_{2}\right\}$ is SNC at this point. If the normal qualification condition

$$
N\left(\bar{x} ; \Omega_{1}\right) \cap\left(-N\left(\bar{x} ; \Omega_{2}\right)\right)=\{0\}
$$

is satisfied, then we have the inclusion

$$
N\left(\bar{x} ; \Omega_{1} \cap \Omega_{2}\right) \subset N\left(\bar{x} ; \Omega_{1}\right)+N\left(\bar{x} ; \Omega_{2}\right) .
$$

For establishing optimality conditions, the following lemma, which is related to the limiting subdifferential calculus, is very useful.

Lemma 2.2. [11, Theorem 3.36 and Theorem 3.46]

(i) Let $\phi_{i}: X \rightarrow \overline{\mathbb{R}}, i=1,2, \ldots, m, m \geq 2$ be lower semicontinuous around $\bar{x} \in X$, and let all but one of these functions be Lipschitz continuous around $\bar{x}$. Then

$$
\partial\left(\phi_{1}+\phi_{2}+\ldots+\phi_{m}\right)(\bar{x}) \subset \partial \phi_{1}(\bar{x})+\partial \phi_{2}(\bar{x})+\ldots+\partial \phi_{m}(\bar{x}) .
$$

(ii) Let $\phi_{i}: X \rightarrow \overline{\mathbb{R}}, i=1,2, \ldots, m, m \geq 2$ be lower semicontinuous around $\bar{x}$ for $i \in I_{\max }(\bar{x})$ and be upper semicontinuous at $\bar{x}$ for $i \notin I_{\max }(\bar{x})$. Suppose that each $\phi_{i}, i=1, \ldots, m$, is Lipschitz continuous around $\bar{x}$. Then we have the inclusion

$$
\partial\left(\max \phi_{i}\right)(\bar{x}) \subset \bigcup\left\{\partial\left(\sum_{i \in I_{\max }(\bar{x})} \lambda_{i} \phi_{i}\right)(\bar{x}) \mid\left(\lambda_{1}, \ldots, \lambda_{m}\right) \in \Lambda(\bar{x})\right\},
$$


where the equality holds and the maximum functions are lower regular at $\bar{x}$ if each $\phi_{i}$ is lower regular at this point and sets $I_{\max }(\bar{x})$ and $\Lambda(\bar{x})$ are defined as follows:

$$
\begin{aligned}
& I_{\max }(\bar{x}):=\left\{i \in\{1, \ldots, m\} \mid \phi_{i}(\bar{x})=\left(\max \phi_{i}\right)(\bar{x})\right\}, \\
& \Lambda(\bar{x}):=\left\{\left(\lambda_{1}, \ldots, \lambda_{m}\right) \mid \lambda_{i} \geq 0, \sum_{i=1}^{m} \lambda_{i}=1, \lambda_{i}\left(\phi_{i}(\bar{x})-\left(\max \phi_{i}\right)(\bar{x})\right)=0\right\} .
\end{aligned}
$$

\section{Optimality CONDitions}

In this section, we first establish necessary optimality conditions for locally optimal solutions of problem $(\mathrm{P})$. Then, by introducing concepts of generalized convexity, we show sufficient conditions for such solutions for problem (P). Below, we give the definition of solutions for problem $(\mathrm{P})$.

Definition 3.1. Let $\phi(x):=\max _{k \in K} f_{k}(x), x \in X$. A point $\bar{x} \in F$ is called a locally optimal solution of problem (P) if and only if there is a neighborhood $U$ of $\bar{x}$ such that

$$
\phi(\bar{x}) \leq \phi(x) \text { for all } x \in U \cap F .
$$

Now, denote by $\mathbb{R}^{(T)}$ the linear space of generalized finite sequences $\lambda=\left(\lambda_{t}\right)_{t \in T}$ such that $\lambda_{t} \in \mathbb{R}$ for each $t \in T$ but only finitely many $\lambda_{t}$ differ from zero. In other words,

$$
\mathbb{R}^{(T)}:=\left\{\lambda=\left(\lambda_{t}\right)_{t \in T} \mid \lambda_{t}=0 \text { for all } t \in T \text { but only finitely many } \lambda_{t} \neq 0\right\} .
$$

The nonnegative cone of $\mathbb{R}^{(T)}$ is denoted by

$$
\mathbb{R}_{+}^{(T)}:=\left\{\lambda=\left(\lambda_{t}\right)_{t \in T} \in \mathbb{R}^{(T)} \mid \lambda_{t} \geq 0, t \in T\right\} .
$$

With $\lambda \in \mathbb{R}^{(T)}$, its supporting set, denoted by $T(\lambda):=\left\{t \in T \mid \lambda_{t} \neq 0\right\}$, is a finite subset of $T$.

Definition 3.2. One says that the constraint qualification (CQ) is satisfied at $\bar{x} \in \Omega$ if there do not exist $\lambda_{t} \geq 0, t \in T(\lambda)$, such that

$$
0 \in \sum_{t \in T} \lambda_{t} \partial g_{t}(\bar{x})+N(\bar{x} ; \Omega)
$$

Now, we establish Karush-Kuhn-Tucker necessary conditions for locally optimal solutions of problem $(\mathrm{P})$.

Theorem 3.1. Let the (CQ) be satisfied at $\bar{x} \in \Omega$. If $\bar{x}$ is a locally optimal solution of problem (P), then there exist $\alpha_{k} \geq 0, k \in K$ with $\sum_{k \in K} \alpha_{k}=1, \lambda \in \mathbb{R}_{+}^{(T)}$, such that

$$
\begin{aligned}
& 0 \in \sum_{k \in K} \alpha_{k} \partial f_{k}(\bar{x})+\sum_{t \in T} \lambda_{t} \partial g_{t}(\bar{x})+N(\bar{x} ; \Omega), \\
& \alpha_{k}\left(f_{k}(\bar{x})-\max _{k \in K} f_{k}(\bar{x})\right)=0, k \in K, \\
& g_{t}(\bar{x})=0, t \in T(\lambda) .
\end{aligned}
$$

Proof. Let $\bar{x}$ be a locally optimal solution of problem (P), equivalently, $\bar{x}$ is a locally optimal solution of the following unconstrained optimization problem

$$
\min _{x \in X} \phi(x)+\delta(x ; F),
$$


where $\phi(x):=\max _{k \in K} f_{k}(x)$. Invoking now the nonsmooth version of Fermat's rule (2.2) to problem (3.2), we have

$$
0 \in \partial(\phi+\delta(\cdot ; F))(\bar{x})
$$

Since the function $\delta(\cdot ; F)$ is l.s.c around $\bar{x}$ and the function $\phi$ is Lipschitz continuous around $\bar{x}$, it follows from the sum rule (2.3) applied to (3.3) and from the relation in (2.1) that

$$
0 \in \partial \phi(\bar{x})+N(\bar{x} ; F) .
$$

Applying further the formula for the limiting subdifferential of maximum functions and the limiting subdifferential sum rule for Lipschitz functions (Lemma 2.2), we have

$$
\begin{aligned}
\partial \phi(\bar{x}) & =\partial\left(\max _{k \in K} f_{k}\right)(\bar{x}) \\
& \subset\left\{\sum_{k \in K(\bar{x})} \alpha_{k} \partial f_{k}(\bar{x}) \mid \alpha_{k} \geq 0, \alpha_{k}\left(f_{k}(\bar{x})-\max _{k \in K} f_{k}(\bar{x})\right)=0, k \in K(\bar{x}), \sum_{k \in K(\bar{x})} \alpha_{k}=1\right\},
\end{aligned}
$$

where $K(\bar{x}):=\left\{k \in K \mid f_{k}(\bar{x})=\phi(\bar{x})\right\} \neq \emptyset$.

On the other hand, by letting

$$
\tilde{\Omega}:=\left\{x \in X \mid g_{t}(x) \leq 0, t \in T\right\},
$$

we have $F:=\tilde{\Omega} \cap \Omega$. The (CQ) being fulfilled at $\bar{x}$ entails that there do not exist $\lambda_{t} \geq 0, t \in T(\lambda)$ such that

$$
0 \in \sum_{t \in T} \lambda_{t} \partial g_{t}(\bar{x})
$$

Hence, we conclude by [12, Exercise 3.74$]$ that

$$
N(\bar{x} ; \tilde{\Omega}) \subset\left\{\sum_{t \in T} \lambda_{t} \partial g_{t}(\bar{x}) \mid \lambda_{t} \geq 0, t \in T(\lambda)\right\} .
$$

As the (CQ) is satisfied at $\bar{x}$, we obtain $g_{t}(\bar{x})=0, t \in T(\lambda)$. If it is not valid, then $g_{t}(\bar{x})<0$, $t \in T(\lambda)$, such that $\bar{x}$ is an interior point of feasible set, and there exist $\lambda_{t} \geq 0, t \in T(\lambda)$ hold $0 \in$ $\sum_{t \in T} \lambda_{t} \partial g_{t}(\bar{x})+N(\bar{x} ; \Omega)$. This contradicts the (CQ) condition. Due to the fact that $\Omega$ is assumed to be SNC at $\bar{x}$, we apply Lemma 2.1 to obtain

$$
N(\bar{x} ; F)=N(\bar{x} ; \tilde{\Omega} \cap \Omega) \subset N(\bar{x} ; \tilde{\Omega})+N(\bar{x} ; \Omega) .
$$

It follows from (3.4), (3.5), and (3.6) that

$$
\begin{aligned}
0 \in & \left\{\sum_{k \in K(\bar{x})} \alpha_{k} \partial f_{k}(\bar{x}) \mid \alpha_{k} \geq 0, \alpha_{k}\left(f_{k}(\bar{x})-\max _{k \in K} f_{k}(\bar{x})\right)=0, k \in K(\bar{x}), \sum_{k \in K(\bar{x})} \alpha_{k}=1\right\} \\
& +\left\{\sum_{t \in T(\lambda)} \lambda_{t} \partial g_{t}(\bar{x}) \mid \lambda_{t} \geq 0, t \in T(\lambda)\right\}+N(\bar{x} ; \Omega) .
\end{aligned}
$$

Since $\lambda \in \mathbb{R}_{+}^{(T)}$, put $\lambda_{t}:=0$ for $t \in T \backslash T(\lambda), \alpha_{k}:=0$ for $k \in K \backslash K(\bar{x})$. Now, it is clear that (3.7) implies (3.1), and that completes the proof. 
The following example shows that the conclusion of Theorem 3.1 may fail to hold if the (CQ) is not satisfied at the point under consideration. In other words, the following example asserts the importance of the (CQ) imposed in Theorem 3.1. This means that the (CQ) in Theorem 3.1 is essential.

Example 3.1. Let $f: \mathbb{R} \rightarrow \mathbb{R}^{2}$ be defined by $f:=\left(f_{1}, f_{2}\right)$, where $f_{1}(x):=f_{2}(x):=x-x^{3}$, and let $g_{t}:=\mathbb{R} \rightarrow \mathbb{R}$ be given by $g_{t}(x)=t x^{2}, x \in \mathbb{R}, t \in T:=[1,2]$. We consider problem (P) with $K:=\{1,2\}$ and $\Omega:=(-\infty, 0]$. Then $F:=\{0\}$ and thus, $\bar{x}:=0$ is a locally optimal solution of $(\mathrm{P})$. Since $N(\bar{x} ; \Omega):=[0, \infty)$ and $\partial g_{t}(\bar{x})=0$ for all $t \in T$, we have

$$
0 \in \sum_{t \in T} \lambda_{t} \partial g_{t}(\bar{x})+N(\bar{x} ; \Omega)
$$

where $\lambda_{t} \geq 0, t \in T(\lambda)$. Hence the (CQ) is not satisfied at $\bar{x}$. Actually, Karush-Kuhn-Tucker conditions (3.1) fail to hold by choosing $\alpha \in \mathbb{R}_{+}^{2}$ with $\alpha_{1}+\alpha_{2}=1$.

Now, we introduce the concept of the generalized convexity to obtain sufficient conditions for (globally) optimal solutions of problem (P). This is inspired by [10, 13, 14, 15].

Definition 3.3. We say that $\left(f, g_{T}\right)$ is generalized convex on $\Omega$ at $\bar{x} \in \Omega$ if, for any $x \in \Omega$, $\xi_{k} \in \partial f_{k}(\bar{x}), k \in K$ and any $\eta_{t} \in \partial g_{t}(\bar{x}), t \in T$, there exists $v \in N(\bar{x} ; \Omega)^{\circ}$ such that

$$
\begin{gathered}
f_{k}(x)-f_{k}(\bar{x}) \geq\left\langle\xi_{k}, v\right\rangle, k \in K, \\
g_{t}(x)-g_{t}(\bar{x}) \geq\left\langle\eta_{t}, v\right\rangle, t \in T .
\end{gathered}
$$

The following theorem describes sufficient optimality conditions for (globally) optimal solutions of problem $(\mathrm{P})$.

Theorem 3.2. Let $\bar{x} \in F$ satisfy Karush-Kuhn-Tucker conditions (3.1). If $\left(f, g_{T}\right)$ is generalized convex on $\Omega$ at $\bar{x}$, then $\bar{x}$ is a (globally) optimal solution of problem $(\mathrm{P})$.

Proof. Let $\phi(x):=\max _{k \in K} f_{k}(x)$. Since $\bar{x} \in F$ satisfies condition (3.1), there exist $\alpha_{k} \geq 0, k \in K$ with $\sum_{k \in K} \alpha_{k}=1, \lambda \in \mathbb{R}_{+}^{(T)}$, and $\xi_{k} \in \partial f_{k}(\bar{x}), k \in K, \eta_{t} \in \partial g_{t}(\bar{x}), t \in T$, such that

$$
\begin{aligned}
& -\left(\sum_{k \in K} \alpha_{k} \xi_{k}+\sum_{t \in T} \lambda_{t} \eta_{t}\right) \in N(\bar{x} ; \Omega), \\
& \alpha_{k}\left(f_{k}(\bar{x})-\max _{k \in K} f_{k}(\bar{x})\right)=0, k \in K, \\
& g_{t}(\bar{x})=0, t \in T(\lambda) .
\end{aligned}
$$

Assume to the contrary that $\bar{x}$ is not a globally optimal solution of problem (P). Then there is $\hat{x} \in F$ such that

$$
\phi(\bar{x})>\phi(\hat{x}) .
$$

By the generalized convexity of $\left(f, g_{T}\right)$ on $\Omega$ at $\bar{x}$, we deduce from (3.8) that, for such $\hat{x}$, there is $v \in N(\bar{x} ; \Omega)^{\circ}$ such that

$$
\begin{aligned}
0 & \leq \sum_{k \in K} \alpha_{k}\left\langle\xi_{k}, v\right\rangle+\sum_{t \in T} \lambda_{t}\left\langle\eta_{t}, v\right\rangle \\
& \leq \sum_{k \in K} \alpha_{k}\left(f_{k}(\hat{x})-f_{k}(\bar{x})\right)+\sum_{t \in T} \lambda_{t}\left(g_{t}(\hat{x})-g_{t}(\bar{x})\right) .
\end{aligned}
$$


Hence,

$$
\sum_{k \in K} \alpha_{k} f_{k}(\bar{x}) \leq \sum_{k \in K} \alpha_{k} f_{k}(\hat{x})+\sum_{t \in T} \lambda_{t}\left(g_{t}(\hat{x})-g_{t}(\bar{x})\right)
$$

In addition, $\hat{x} \in F$ which yileds $\lambda_{t} g_{t}(\hat{x}) \leq 0$. So, we obtain by (3.10), (3.12) that

$$
\sum_{k \in K} \alpha_{k} f_{k}(\bar{x}) \leq \sum_{k \in K} \alpha_{k} f_{k}(\hat{x})
$$

On the other hand, by (3.9), it holds that

$$
\sum_{k \in K} \alpha_{k} \phi(\bar{x})=\sum_{k \in K} \alpha_{k} f_{k}(\bar{x}) \leq \sum_{k \in K} \alpha_{k} \phi(\hat{x})
$$

This implies that

$$
\phi(\bar{x}) \leq \phi(\hat{x}) .
$$

Obviously, (3.13) contradicts (3.11), which completes the proof.

\section{Duality Relations}

In this section, we formulate a dual problem for problem (P), and explore weak, strong, and converse-like duality relations between problem (P) and its dual.

In connection with the semi-infinite minimax optimization problem $(\mathrm{P})$, we consider a dual problem in the following form:

$$
\max \left\{\tilde{\phi}(z, \alpha, \lambda):=\phi(z)+\sum_{t \in T} \lambda_{t} g_{t}(z) \mid(z, \alpha, \lambda) \in F_{D}\right\},
$$

where $\phi(z):=\max _{k \in K} f_{k}(z)$, and the constraint set $F_{D}$ is defined by

$$
\begin{array}{r}
F_{D}:=\left\{(z, \alpha, \lambda) \in \Omega \times \mathbb{R}_{+}^{l} \times \mathbb{R}_{+}^{(T)} \mid 0 \in \sum_{k \in K} \alpha_{k} \partial f_{k}(z)+\sum_{t \in T} \lambda_{t} \partial g_{t}(z)+N(z ; \Omega),\right. \\
\left.\alpha_{k}\left(f_{k}(z)-\phi(z)\right)=0, k \in K, \sum_{k \in K} \alpha_{k}=1\right\} .
\end{array}
$$

Definition 4.1. A point $(\bar{z}, \bar{\alpha}, \bar{\lambda}) \in F_{D}$ is called a globally optimal solution of problem (D) if and only if

$$
\tilde{\phi}(\bar{z}, \bar{\alpha}, \bar{\lambda}) \geq \tilde{\phi}(z, \alpha, \lambda), \forall(z, \alpha, \lambda) \in F_{D}
$$

The next theorem shows weak duality relations between (P) and (D).

Theorem 4.1. Let $x \in F$, and let $(z, \alpha, \lambda) \in F_{D}$. If $\left(f, g_{T}\right)$ is generalized convex on $\Omega$ at $z$, then

$$
\phi(x) \geq \tilde{\phi}(z, \alpha, \lambda)
$$

where $\phi(x):=\max _{k \in K} f_{k}(x)$. 
Proof. Since $(z, \alpha, \lambda) \in F_{D}$, there exist $\alpha:=\left(\alpha_{1}, \ldots, \alpha_{l}\right) \in \mathbb{R}_{+}^{l}$ with $\sum_{k \in K} \alpha_{k}=1, \lambda \in \mathbb{R}_{+}^{(T)}$, $\xi_{k} \in \partial f_{k}(z), k \in K, \eta_{t} \in \partial g_{t}(z), t \in T$, such that

$$
\begin{aligned}
& -\left(\sum_{k \in K} \alpha_{k} \xi_{k}+\sum_{t \in T} \lambda_{t} \eta_{t}\right) \in N(z ; \Omega), \\
& \alpha_{k}\left(f_{k}(z)-\phi(z)\right)=0, k \in K .
\end{aligned}
$$

By the generalized convexity of $\left(f, g_{T}\right)$ on $\Omega$ at $z$, there exists $v \in N(z ; \Omega)^{\circ}$. Due to the definition of polar cones, it follows from (4.1) and the relation $v \in N(z ; \Omega)^{\circ}$ that

$$
\begin{aligned}
0 & \leq \sum_{k \in K} \alpha_{k}\left\langle\xi_{k}, v\right\rangle+\sum_{t \in T} \lambda_{t}\left\langle\eta_{t}, v\right\rangle \\
& \leq \sum_{k \in K} \alpha_{k}\left(f_{k}(x)-f_{k}(z)\right)+\sum_{t \in T} \lambda_{t}\left(g_{t}(x)-g_{t}(z)\right) .
\end{aligned}
$$

In addition, due to $\lambda \in \mathbb{R}_{+}^{(T)}$, and $x \in F$, we have $\sum_{t \in T} \lambda_{t} g_{t}(x) \leq 0$. Taking now (4.2), above inequality yields

$$
\begin{aligned}
\phi(z)+\sum_{t \in T} \lambda_{t} g_{t}(z) & =\sum_{k \in K} \alpha_{k} f_{k}(z)+\sum_{t \in T} \lambda_{t} g_{t}(z) \\
& \leq \sum_{k \in K} \alpha_{k} f_{k}(x) \\
& =\phi(x) .
\end{aligned}
$$

This completes the proof.

The forthcoming theorem describes strong duality relations between (P) and (D).

Theorem 4.2. Let $\bar{x} \in \Omega$ be a locally optimal solution of problem (P) such that the (CQ) is satisfied at this point. Then there exists $(\bar{\alpha}, \bar{\lambda}) \in \mathbb{R}_{+}^{l} \times \mathbb{R}_{+}^{(T)}$ such that $(\bar{x}, \bar{\alpha}, \bar{\lambda}) \in F_{D}$ and

$$
\phi(\bar{x})=\tilde{\phi}(\bar{x}, \bar{\alpha}, \bar{\lambda}) \text {, }
$$

where $\phi(x):=\max _{k \in K} f_{k}(x)$. Furthermore, if $\left(f, g_{T}\right)$ is generalized convex on $\Omega$ at any $z \in \Omega$, then $(\bar{x}, \bar{\alpha}, \bar{\lambda})$ is an optimal solution of problem (D).

Proof. Applying Theorem 3.1, we find $\bar{\alpha}:=\left(\bar{\alpha}_{1}, \ldots, \bar{\alpha}_{l}\right) \in \mathbb{R}_{+}^{l}$ with $\sum_{k \in K} \bar{\alpha}_{k}=1$, and $\bar{\lambda}:=$ $\left(\bar{\lambda}_{t}\right)_{t \in T} \in \mathbb{R}_{+}^{(T)}$, such that

$$
\begin{aligned}
& 0 \in \sum_{k \in K} \bar{\alpha}_{k} \partial f_{k}(\bar{x})+\sum_{t \in T} \bar{\lambda}_{t} \partial g_{t}(\bar{x})+N(\bar{x} ; \Omega), \\
& \bar{\alpha}_{k}\left(f_{k}(\bar{x})-\phi(\bar{x})\right)=0, k \in K, \\
& g_{t}(\bar{x})=0, t \in T(\lambda) .
\end{aligned}
$$

Hence $\sum_{t \in T} \bar{\lambda}_{t} g_{t}(\bar{x})=0$, we conclude that $\phi(\bar{x})=\tilde{\phi}(\bar{x}, \bar{\alpha}, \bar{\lambda})$. Clearly, $(\bar{x}, \bar{\alpha}, \bar{\lambda}) \in F_{D}$. If $\left(f, g_{T}\right)$ is generalized convex on $\Omega$ at any $z \in \Omega$, then by Theorem 4.1, we obtain

$$
\tilde{\phi}(\bar{x}, \bar{\alpha}, \bar{\lambda})=\phi(\bar{x}) \geq \tilde{\phi}(z, \alpha, \lambda)
$$

for any $(z, \alpha, \lambda) \in F_{D}$. Hence, $(\bar{x}, \bar{\alpha}, \bar{\lambda})$ is an optimal solution of problem (D).

We close this section by proving the converse-like duality relation between (P) and (D). 
Theorem 4.3. Let $(\bar{x}, \bar{\alpha}, \bar{\lambda}) \in F_{D}$ be such that $\phi(\bar{x})=\tilde{\phi}(\bar{x}, \bar{\alpha}, \bar{\lambda})$ with $\phi(\bar{x}):=\max _{k \in K} f_{k}(\bar{x})$. If $\bar{x} \in F$ and $\left(f, g_{T}\right)$ is generalized convex on $\Omega$ at $\bar{x}$, then $\bar{x}$ is a globally optimal solution of problem $(\mathrm{P})$.

Proof. Since $(\bar{x}, \bar{\alpha}, \bar{\lambda}) \in F_{D}$, there exist $\bar{\alpha}:=\left(\bar{\alpha}_{1}, \ldots, \bar{\alpha}_{l}\right) \in \mathbb{R}_{+}^{l}$ with $\sum_{k \in K} \bar{\alpha}_{k}=1, \bar{\lambda}:=\left(\bar{\lambda}_{t}\right)_{t \in T} \in$ $\mathbb{R}_{+}^{(T)}, \bar{\xi}_{k} \in \partial f_{k}(\bar{x}), k \in K, \bar{\eta}_{t} \in \partial g_{t}(\bar{x}), t \in T$, such that

$$
\begin{aligned}
& -\left(\sum_{k \in K} \bar{\alpha}_{k} \bar{\xi}_{k}+\sum_{t \in T} \bar{\lambda} \bar{\eta}_{t}\right) \in N(\bar{x} ; \Omega), \\
& \bar{\alpha}_{k}\left(f_{k}(\bar{x})-\phi(\bar{x})\right)=0, k \in K .
\end{aligned}
$$

Let $\bar{x} \in F$. We have $g_{t}(\bar{x}) \leq 0$ for $t \in T$. Hence, $\sum_{t \in T} \bar{\lambda}_{t} g_{t}(\bar{x}) \leq 0$, which together with $\phi(\bar{x})=$ $\tilde{\phi}(\bar{x}, \bar{\alpha}, \bar{\lambda})$ yields $g_{t}(\bar{x})=0$ for all $t \in T(\lambda)$, i.e., $\bar{\lambda} \in \mathbb{R}_{+}^{(T)}$. This together with (4.3) and (4.4) confirms that $\bar{x}$ satisfies condition (3.1). To finish the proof, it remains to apply Theorem 3.2.

\section{Applications to Multiobjective Optimization Problems}

In this section, we establish necessary and sufficient conditions for a weak Pareto solution of (MP) by employing optimality conditions obtained by (P) in Section 3.

Consider the following semi-infinite multiobjective optimization problem:

$$
\min _{\mathbb{R}_{+}^{l}}\left\{f(x):=\left(f_{1}(x), \ldots, f_{l}(x)\right) \mid x \in F\right\},
$$

where $F$ is same as the feasible set of problem $(\mathrm{P})$.

Definition 5.1. A point $\bar{x} \in F$ is said to be a weak Pareto solution of problem (MP) if and only if

$$
f(x)-f(\bar{x}) \notin-\operatorname{int} \mathbb{R}_{+}^{l}, \forall x \in F .
$$

The following result is a Karush-Kuhn-Tucker (KKT) necessary condition for a weak Pareto solution of problem (MP).

Theorem 5.1. Let the (CQ) be satisfied at $\bar{x} \in \Omega$. If $\bar{x}$ is a weak Pareto solution of problem (MP), then there exist $\alpha:=\left(\alpha_{1}, \ldots, \alpha_{l}\right) \in \mathbb{R}_{+}^{l}$ with $\sum_{k \in K} \alpha_{k}=1$, and $\lambda \in \mathbb{R}_{+}^{(T)}$, such that

$$
\begin{aligned}
& 0 \in \sum_{k \in K} \alpha_{k} \partial f_{k}(\bar{x})+\sum_{t \in T} \lambda_{t} \partial g_{t}(\bar{x})+N(\bar{x} ; \Omega), \\
& g_{t}(\bar{x})=0, t \in T(\lambda) .
\end{aligned}
$$

Proof. Let $\bar{x}$ be a weak Pareto solution of problem (MP) and let

$$
\widehat{f}_{k}(x):=f_{k}(x)-f_{k}(\bar{x}), k \in K, x \in X .
$$

By [10, Theorem 5.1], we can show that $\bar{x}$ is an optimal solution of the minimax optimization problem

$$
\min _{x \in F} \max _{k \in K} \widehat{f}_{k}(x)
$$


So, we can employ the KKT condition in Theorem 3.1. We find $\alpha \in \mathbb{R}_{+}^{l}$ with $\sum_{k \in K} \alpha_{k}=1$, and $\lambda \in \mathbb{R}_{+}^{(T)}$, such that

$$
\begin{aligned}
& 0 \in \sum_{k \in K} \alpha_{k} \partial f_{k}(\bar{x})+\sum_{t \in T} \lambda_{t} \partial g_{t}(\bar{x})+N(\bar{x} ; \Omega), \\
& g_{t}(\bar{x})=0, t \in T(\lambda) .
\end{aligned}
$$

Thus, the proof is complete.

The last theorem of this work describes sufficient optimality conditions for a weak Pareto solution of problem (MP).

Theorem 5.2. Let $\bar{x} \in F$ satisfy condition (5.1). If $\left(f, g_{T}\right)$ is generalized convex on $\Omega$ at $\bar{x}$, then $\bar{x}$ is a weak Pareto solution of problem (MP).

Proof. Set

$$
\widehat{f}_{k}(x):=f_{k}(x)-f_{k}(\bar{x}), k \in K, x \in X .
$$

Let $\bar{x} \in F$ satisfy condition (5.1). It is clear that the following condition holds

$$
\alpha_{k}\left(\widehat{f}_{k}(\bar{x})-\max _{k \in K} \widehat{f}_{k}(\bar{x})\right)=0, k \in K
$$

Let $\widehat{f}:=\left(\widehat{f}_{1}, \ldots, \widehat{f}_{l}\right)$. Since $\left(f, g_{T}\right)$ is generalized convex on $\Omega$ at $\bar{x}$, it follows that $\left(\widehat{f}, g_{T}\right)$ is generalized convex on $\Omega$ at this point as well. Thus, we apply the sufficient criteria in Theorem 3.2 to conclude that $\bar{x}$ is a globally optimal solution of the minimax optimization problem

$$
\min _{x \in F} \max _{k \in K} \widehat{f}_{k}(x)
$$

It means that

$$
\widehat{\phi}(\bar{x}) \leq \widehat{\phi}(x), \forall x \in F,
$$

where $\widehat{\phi}(x):=\max _{k \in K} \widehat{f}_{k}(x)$. In other words, we obtain

$$
0 \leq \max _{k \in K}\left\{f_{k}(x)-f_{k}(\bar{x})\right\}, \forall x \in F .
$$

This inequality ensures that $\bar{x}$ is a weak Pareto solution of problem (MP).

\section{REFERENCES}

[1] G. Caristi, M. Ferrara and A. Stefanescu, Semi-infinite multiobjective programming with generalized invexity, Math. Rep. 12 (2010), 217-233.

[2] M. A. Goberna, M. A. López, Linear Semi-infinite Optimization, Wiley, Chichester, 1998.

[3] T.D. Chuong, J.-C. Yao, Sufficient conditions for pseudo-Lipschitz property in convex semi-infinite vector optimization problems, Nonlinear Anal. 71 (2009), 6312-6322.

[4] T.D. Chuong, N.Q. Huy, J.-C. Yao, Stability of semi-infinite vector optimization problems under functional perturbations, J. Glob. Optim. 45 (2009), 583-595.

[5] T.Q. Son, J.J. Strodiot, V.H. Nguyen, $\varepsilon$-Optimality and $\varepsilon$-Lagrangian duality for a nonconvex programming problem with an infinite number of constraints, J. Optim. Theory Appl. 141 (2009), 389-409.

[6] T.Q. Son, D.S. Kim, N.N. Tam, Weak stability and strong duality of a class of nonconvex infinite programs via augmented Lagrangian, J. Glob. Optim. 53 (2012), 165-184.

[7] L.-N. Zhong, Y.-F. Jin, Optimality conditions for minimax optimization problems with an infinite number of constraints and related applications, Acta Math. Appl. Sin. Engl. Ser. 37 (2021), 251-263. 
[8] J. Bram, The Lagrange multiplier theorem for maxmin with several constraints, SIAM J. Appl. Math. 14 (1966), 665-667.

[9] C. R. Bector, S. Chandra, V. Kumar, Duality for a class of minmax and inexact programming problem, J. Math. Anal. Appl. 186(3) (1994), 735-746.

[10] T.D. Chuong, D.S. Kim, Nondifferentiable minimax programming problems with applications, Ann. Oper. Res. 251 (2017), 73-87.

[11] B.S. Mordukhovich, Variational Analysis and Generalized Differentiation, I: Basic Theory, Springer Verlag, 2006.

[12] B.S. Mordukhovich, Variational Analysis and Applications, Springer Monographs in Mathematics, 2018.

[13] T.D. Chuong, D.S. Kim, Nonsmooth semi-infinite multiobjective optimization problems, J. Optim. Theory Appl. 160 (2014), 748-762.

[14] T.D. Chuong, D.S. Kim, A class of nonsmooth fractional multiobjective optimization problems, Ann. Oper. Res. 244 (2016), 367-383.

[15] Z. Hong, L.G. Jiao, D.S. Kim, On a class of nonsmooth fractional robust multi-objective optimization problems. Part I: optimality conditions, Appl. Set-Valued Anal. Optim. 2 (2020), 109-121. 